\title{
Inflammatory Reactions to Red Tattoo Inks Three cases highlighting an emerging problem
}

*Ricardo Ruiz-Villaverde, ${ }^{1}$ Pablo Fernandez-Crehuet, ${ }^{2}$ Paula Aguayo-Carreras, ${ }^{3}$ Jose L. Hernandez-Centeno, ${ }^{2}$ Carlos Cuenca-Barrales ${ }^{3}$

$$
\begin{aligned}
& \text { ردود الفعل الالتهابية لأحبار الوشم الاحمر } \\
& \text { ثلاث حالات تسلط الضوء على مشكلة مستجدة }
\end{aligned}
$$

ريكاردو رويز-فيلافيردي، بابلو فرنانديز-كراويت، باولا أغوايو-كاريراس، خوسيه لويس هيرنانديز-سينتينو، كارلوس كوينكا-باراليس

ABSTRACT: In recent years, tattoos have become more commonplace. However, this can result in various inflammatory processes, the management of which can be challenging in daily clinical practice. Tattoo-related inflammatory reactions can comprise different patterns, including acute and immediate reactions, foreign body granulomas, sarcoid granulomas, isomorphic lesions, allergic contact dermatitis and photosensitivity. We report three cases who were referred to the Dermatology Outpatient Clinic of the Hospital Universitario San Cecilio, Granada, Spain, in 2017 with various skin reactions in the red-ink areas of their tattoos. Screening was performed for infectious diseases like atypical mycobacterial infections and systemic processes such as sarcoidosis. A good therapeutic response was achieved in all cases. An adequate differential diagnosis is essential for the therapeutic management of this emerging health problem.

Keywords: Non-Therapeutic Body Modification; Tattooing, adverse effects; Inks; Foreign Body Reaction; Inflammation; Case Report; Spain.

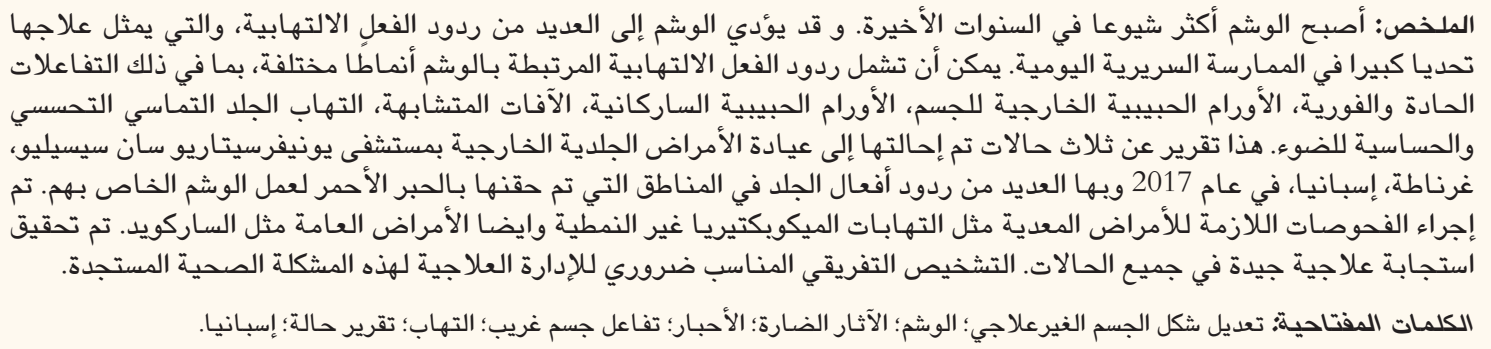

\section{$\mathrm{T}$} He popularity of tattoos has increased in recent years. ${ }^{1}$ Inflammatory reactions to red tattoo inks may be due to the pigment itself, auxiliary ingredients in the ink (i.e. preservatives, binders or thickeners) or impurities generated during the manufacturing process. ${ }^{2}$ Nowadays, $80 \%$ of the colour pigments used in tattoo inks are synthetic azo or polycyclic compounds which enhance the brightness, stability and duration of the tattoo. ${ }^{3}$

Most inflammatory reactions to red tattoo inks occur as a response to the red pigment and can have hyperkeratotic, nodular, plaque, exudative or ulcerative manifestations. ${ }^{4}$ Such reactions may have a latency period varying from weeks to months or even years after the tattoo is inked. ${ }^{2,5}$ The majority of such reactions usually occur in tattoos located on the extremities, possibly due to their greater exposure to ultraviolet (UV) radiation, and result in intense pruritus. ${ }^{6}$ We present three patients who developed inflammatory reactions to red tattoo inks and discuss the potential differential diagnoses of such reactions.

\section{Case One}

A 38-year-old female patient was referred to the Dermatology Outpatient Clinic of the Hospital Universitario San Cecilio, Granada, Spain, in 2017. She had gotten a tattoo on the right leg seven months previously. However, two weeks after receiving the tattoo, she began to develop hyperkeratotic papules and nodules on the parts of the tattoo impregnated with red ink [Figure 1]. She reported no relevant medical history, apart from being allergic to sulphonamides. An ultrasound revealed a poorly demarcated hypoechoic epidermal tumour of variable echogenicity without artefacts. There was evidence of abundant intralesional vascularisation in Doppler mode. All complementary tests and investigations were normal, including a complete blood count $(\mathrm{CBC})$, biochemistry panel, thyroid hormone and angiotensin-converting enzyme (ACE) levels, a chest $\mathrm{X}$-ray and an autoimmunity study. 


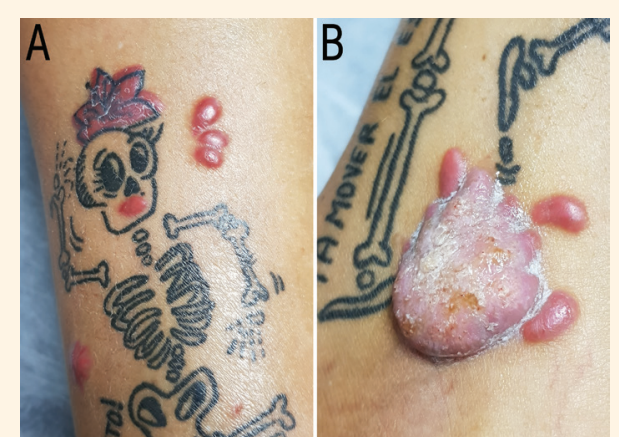

Figure 1: Clinical photographs of the right leg of a 38-yearold female patient (case one) showing an inflammatory reaction to the red ink in a skeleton tattoo.

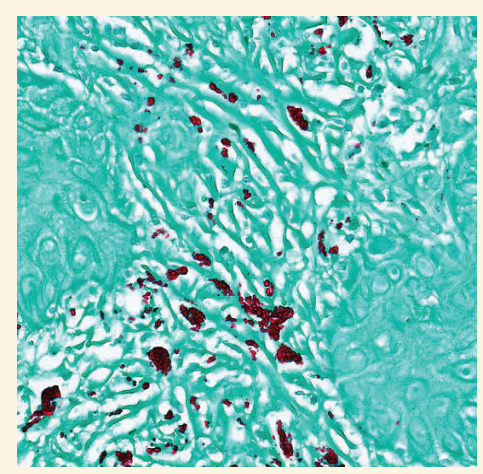

Figure 2: Ziehl-Neelsen stain at x40 magnification showing the enhancement of red pigment in tattoo ink.

A histological examination of the lesion confirmed an intense lymphohistiocytic inflammatory reaction. A Ziehl-Neelsen stain demonstrated the presence of multinucleated foreign body giant cells, with an absence of fungal structures and acid-alcohol-resistant bacilli. The red pigment of the tattoo was curiously enhanced [Figure 2]. A polymerase chain reaction (PCR) analysis for Mycobacterium tuberculosis was also negative. Initially, the patient was treated with topical mometasone furoate for six weeks to promote occlusive healing. Unfortunately, this was not successful. Subsequently, she was prescribed $300 \mathrm{mg} /$ day of allopurinol and $100 \mathrm{mg} /$ day of doxycycline, to which she demonstrated a good therapeutic response after two months.

\section{Case Two}

A 34-year-old female patient was referred to the Dermatology Outpatient Clinic of the Hospital Universitario San Cecilio, Granada, in 2017. She had an indurated erythematous painful nodule with welldefined borders on a six-month-old back tattoo. The lesion was confined to the areas of red pigmentation [Figure 3]. The patient reported a history of malaise,

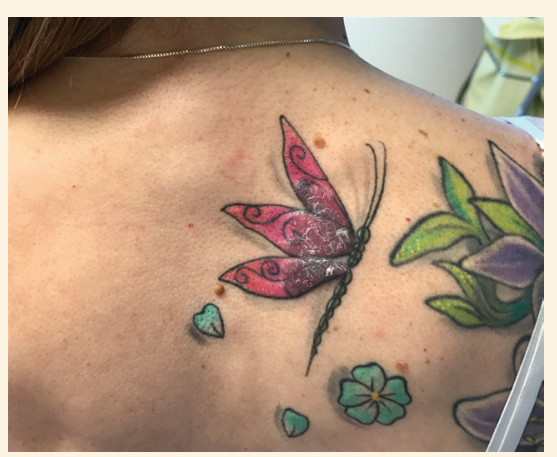

Figure 3: Clinical photograph of the back of a 34-year-old female patient (case two) showing an inflammatory reaction to the red ink in a butterfly tattoo.

anorexia and weight loss over the previous month. Complementary tests and investigations-including a $\mathrm{CBC}$, biochemistry panel, thyroid hormone and ACE levels, chest X-ray and PCR analysis for M. tuberculosis-were all normal.

A punch biopsy was obtained from the indurated area of the tattoo. A histological examination of the sample revealed dense subepithelial lymphoid infiltration, consisting of lymphocytes and histiocytes without germinal centres, eosinophils, large cells or granulomas. There was an absence of cellular atypia. The patient was initially prescribed topical mometasone furoate for six weeks, without improvement. He was then advised to take $0.5 \mathrm{mg} / \mathrm{kg} /$ day of oral prednisone. A medium response to therapy was noted after two months. However, the patient refused further systemic treatment.

\section{Case Three}

A 35-year-old male was referred to the Dermatology Outpatient Clinic of the Hospital Universitario San Cecilio, Granada, in 2017. He presented with a wartlike lesion located on a three-month-old tattoo on the back of his right wrist. The lesion was confined to the part of the skin tattooed with red ink [Figure 4]. These was no visible evidence of any trauma or injury. Complementary tests and investigations were normal, including a $\mathrm{CBC}$, biochemistry panel, thyroid hormone and ACE levels, chest X-ray and PCR analysis for M. tuberculosis.

A histological examination revealed a granulomatous foreign body reaction, most likely due to the red ink in the tattoo. Following an unsuccessful sixweek course of treatment with topical mometasone furoate, the patient demonstrated a good therapeutic response after three months of therapy with $100 \mathrm{mg} /$ day of doxycycline and $300 \mathrm{mg} /$ day of allopurinol. 


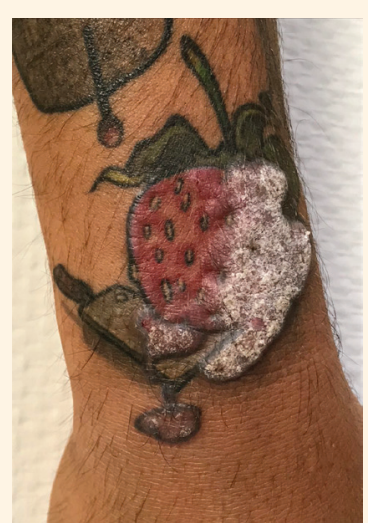

Figure 4: Clinical photograph of the right wrist of a 35-yearold male patient (case three) showing a verrucous reaction to the red ink of a strawberry tattoo.

\section{Discussion}

From a clinical viewpoint, inflammatory reactions can present in different patterns-including acute and immediate reactions after the tattooing process, foreign body granulomas, sarcoid granulomas, isomorphic reactions due to the phenomenon of Koebner, allergic contact dermatitis and photosensitivity. ${ }^{4}$ As such, the diagnosis of an inflammatory reaction to red tattoo ink is not straightforward, since the cutaneous lesions are not specific and the histological findings are not characteristic. Likewise, epicutaneous tests are not informative in most cases. ${ }^{5}$

Even when the original ink used by the tattoo artist is available, the allergen is usually generated as a result of the degradation of the primary components of the ink, rather than because of the ink itself. ${ }^{6}$ For example, azo pigments become chemically unstable when subjected to UV radiation for long periods of time, which causes fragmentation, degradation and diffusion of the ink. In such cases, solar exposure and sunburn considerably increase the risk of developing an inflammatory reaction. ${ }^{6}$ Unfortunately, determining the exact chemical composition of the tattoo inks of the three cases presented in this report was not possible.

A biopsy is essential in cases in which the patient has an inflammatory reaction to red tattoo inks. Although not always conclusive, a biopsy can offer relevant information regarding the type of reaction developed, for example, regarding whether the reaction is granulomatous, pseudolymphomatous, lichenoid, fibrosing or due to a tumour or infection. ${ }^{4,7-9}$ A sarcoid granuloma appearing on a tattoo may be the first or only manifestation of systemic sarcoidosis; this must therefore be excluded from the differential diagnosis as quickly as possible. ${ }^{4}$ In cases of suspected atypical mycobacterial infections, special stains such as a ZiehlNeelsen stain must be used to determine the presence of mycobacteria, as well as a specific PCR analysis. ${ }^{10}$
The clinical course of an inflammatory reaction to red tattoo ink is unpredictable and sometimes disconcerting. Foreign body reactions may resolve spontaneously or the transepidermal elimination of the pigment may take place. ${ }^{11,12}$ For persistent reactions, optimal treatment varies according to the patient's response. Topical potent corticosteroids have been previously reported as a form of occlusive treatment; however, once the lesion has been flattened, the frequency of application should be decreased. ${ }^{5}$ Intralesional corticoids may also be used on a monthly basis. ${ }^{4}$ In terms of systemic treatment, allopurinol is indicated in granulomatous lesions at a dose of 300-600 mg/day for two months. ${ }^{7}$ In addition, effective results have been reported with hydroxychloroquine (200 mg/day for two months). ${ }^{9}$ Oral corticosteroids are recommended in cases with intense pruritus or recurrence, especially if associated with uveitis. ${ }^{4,5}$

Excision of the cutaneous lesion is only useful for patients with small tattoos $(<2 \mathrm{~cm}$ in diameter). However, satisfactory results have been reported for those with larger tattoos when using a dermatome to obtain a graft that covers the entire dermis. ${ }^{13}$ Mohs surgery has also been successfully utilised in more complicated cases. ${ }^{14}$ Laser therapy is another promising therapeutic approach, with a number of successful outcomes and no reports of any significant adverse effects. ${ }^{15}$ In particular, Q-switched potassium titanyl phosphate laser treatment at $532 \mathrm{~nm}$ may be useful in the treatment of red tattoo ink reactions. ${ }^{2}$

\section{Conclusion}

Inflammatory reactions to red tattoo inks are an emerging health problem. Unfortunately, the diagnosis of such reactions can be challenging due to the varied nature of the cutaneous and histological findings. It is therefore important to rule out other differential diagnoses in order to determine an appropriate therapeutic approach.

\section{References}

1. Kluger N. Epidemiology of tattoos in industrialized countries. Curr Probl Dermatol 2015; 48:6-20. doi: 10.1159/000369175.

2. Forbat E, Al-Niaimi F. Patterns of reactions to red pigment tattoo and treatment methods. Dermatol Ther (Heidelb) 2016; 6:13-23. doi: 10.1007/s13555-016-0104-y.

3. de Cuyper C, D'hollander D. Materials used in body art. In: de Cuyper C, Cotapos ML, Eds. Dermatologic Complications with Body Art: Tattoos, piercings and permanent make-up. Berlin, Germany: Springer, 2010. Pp. 13-28. doi: 10.1007/978-3-64203292-9_2.

4. Kluger N. Cutaneous complications related to tattoos: 31 cases from Finland. Dermatology 2017; 233:100-9. doi: 10.1159/ 000468536. 
5. Haber R, Farid S. [Granulomatous tattoo reaction confined to red pigment]. Ann Dermatol Venereol 2016; 143:79-80. doi: 10. 1016/j.annder.2015.09.001.

6. Serup J. How to diagnose and classify tattoo complications in the clinic: A system of distinctive patterns. Curr Probl Dermatol 2017; 52:58-73. doi: 10.1159/000450780.

7. Godinho MM, Aguinaga F, Grynszpan R, Lima VM, Azulay DR, Cuzzi T, et al. Granulomatous reaction to red tattoo pigment treated with allopurinol. J Cosmet Dermatol 2015; 14:241-5. doi: 10.1111 /jocd.12149.

8. Almodovar-Real A, Sánchez-López J, Navarro-Triviño F, Fernández-Pugnaire MA. [Lichenoid reaction to red pigment in a tattoo]. Semergen 2016; 42:e115-17. doi: 10.1016/j.semerg. 2015.12.016.

9. Patrizi A, Raone B, Savoia F, Bacci F, Pileri A, Gurioli C, et al. Tattoo-associated pseudolymphomatous reaction and its successful treatment with hydroxychloroquine. Acta Derm Venereol 2009; 89:327-8. doi: 10.2340/00015555-0639.

10. Wolf R, Wolf D. A tattooed butterfly as a vector of atypical Mycobacteria. J Am Acad Dermatol 2003; 48:S73-4. doi: 10.10 $67 / \mathrm{mjd} .2003 .166$
11. Fang HY, Wu PY, Hsieh HJ. Generalized lichenoid tattoo reaction with spontaneous resolution after skin biopsy. Dermatolo Sinica 2013; 31:38-40. doi: 10.1016/j.dsi.2012.04.003.

12. Høgsberg T, Thomsen BM, Serup J. Histopathology and immune histochemistry of red tattoo reactions: Interface dermatitis is the lead pathology, with increase in T-lymphocytes and Langerhans cells suggesting an allergic pathomechanism. Skin Res Technol 2015; 21:449-58. doi: 10.1111/srt.12213.

13. Mlakar B. Successful removal of hyperkeratotic-lichenoid reaction to red ink tattoo with preservation of the whole tattoo using a skin grafting knife. Acta Dermatovenerol Alp Pannonica Adriat 2015; 24:81-2. doi: 10.15570/actaapa.2015.21.

14. Kim YM, Taylor D, Weiss E, Saleeby ER. Mohs micrographic surgery for a red tattoo reaction. Dermatol Surg 2018; 44:120-2. doi: 10.1097/DSS.0000000000001137.

15. Millán-Cayetano JF, Alegre-Sánchez A, Boixeda P. Treatment of red tattoo reaction using CO2 laser. Lasers Med Sci 2018; 33:1171-3. doi: 10.1007/s10103-017-2229-3. 